\title{
Selective dielectrophoretic manipulation of surface-immobilized DNA molecules
}

\author{
W André Germishuizen ${ }^{1}$, Christoph Wälti ${ }^{2}$, René Wirtz ${ }^{2}$, \\ Michael B Johnston ${ }^{2}$, Michael Pepper ${ }^{2}$, A Giles Davies ${ }^{2}$ and \\ Anton P J Middelberg ${ }^{1}$ \\ ${ }^{1}$ Department of Chemical Engineering, University of Cambridge, Cambridge CB2 3RA, UK \\ ${ }^{2}$ Cavendish Laboratory, University of Cambridge, Cambridge CB3 OHE, UK \\ E-mail: wag22@cam.ac.uk
}

Received 2 October 2002, in final form 15 April 2003

Published 18 June 2003

Online at stacks.iop.org/Nano/14/896

\begin{abstract}
The fabrication of nanoscale molecular devices is becoming increasingly important and research into their fabrication has intensified over the last few years. In particular, the attachment of molecular objects onto various surfaces has attracted considerable attention. Here, we report a multistep surface immobilization procedure, which allows the specific and controlled attachment of very long DNA molecules onto gold electrodes. Further, we report the effect of dielectrophoresis on these surface-bound DNA molecules with respect to amplitude and frequency, and we show that selected surface-immobilized DNA molecules can be manipulated by dielectrophoresis. Finally, we investigated the use of dielectrophoresis in conjunction with the multistep surface immobilization of fluorescently labelled, surface-bound $\lambda$-DNA in a basic data-storage device.
\end{abstract}

(Some figures in this article are in colour only in the electronic version)

\section{Introduction}

With the increasing interest in molecular nanodevices and their fabrication, techniques to handle, manipulate and attach molecules such as DNA to surfaces at specific locations, are attracting considerable research attention [1]. The fabrication process usually involves the specific and controlled attachment of molecules or molecular objects onto a surface. It has been shown that the strong sulfur-metal bond can be used to bind sulfur-functionalized molecules onto metal surfaces simply by immersing the metal surfaces in a solution containing the molecules and, in particular, the attachment of small, thiolated DNA molecules onto gold electrodes has been investigated in detail [2, 3]. However, long DNA molecules assume a random coil conformation. Since the sulfur group at one end of the DNA molecules is unlikely to come into close enough proximity with the gold surface to form a bond, attachment of the molecules using the immersion protocol is then no longer possible.

DNA is a polyelectrolyte, and in solution the phosphate groups on the sugar-phosphate backbone dissociate to form a negatively charged molecule surrounded radially by a cloud of positively charged counterions [4-6]. This makes the DNA highly polarizable, and in an electric field a dipole is induced in the molecule. In a non-uniform electric field, DNA molecules will experience a dielectrophoretic force and an orientational torque as a result of the interaction between the induced DNA dipole and the electric field $[5,7,8]$. The torque and the dielectrophoretic force are a function of the magnitude and frequency of the electric field, the dielectric properties of the medium and the DNA, and the size of the DNA [5, 7]. Dielectrophoresis is a non-contact manipulation technique enabling positioning and orientation of DNA molecules in nanotechnological devices with a high spatial resolution, and is becoming increasingly popular. Together with the dielectrophoretic force, the torque causes the DNA molecules to align parallel to the electric field lines thus elongating them from their equilibrium random coil conformation. However, a detailed and quantitative understanding of the dielectrophoresis of DNA is required to tap the full potential of the technique. Dielectrophoretic manipulation of DNA has been widely studied in the past 

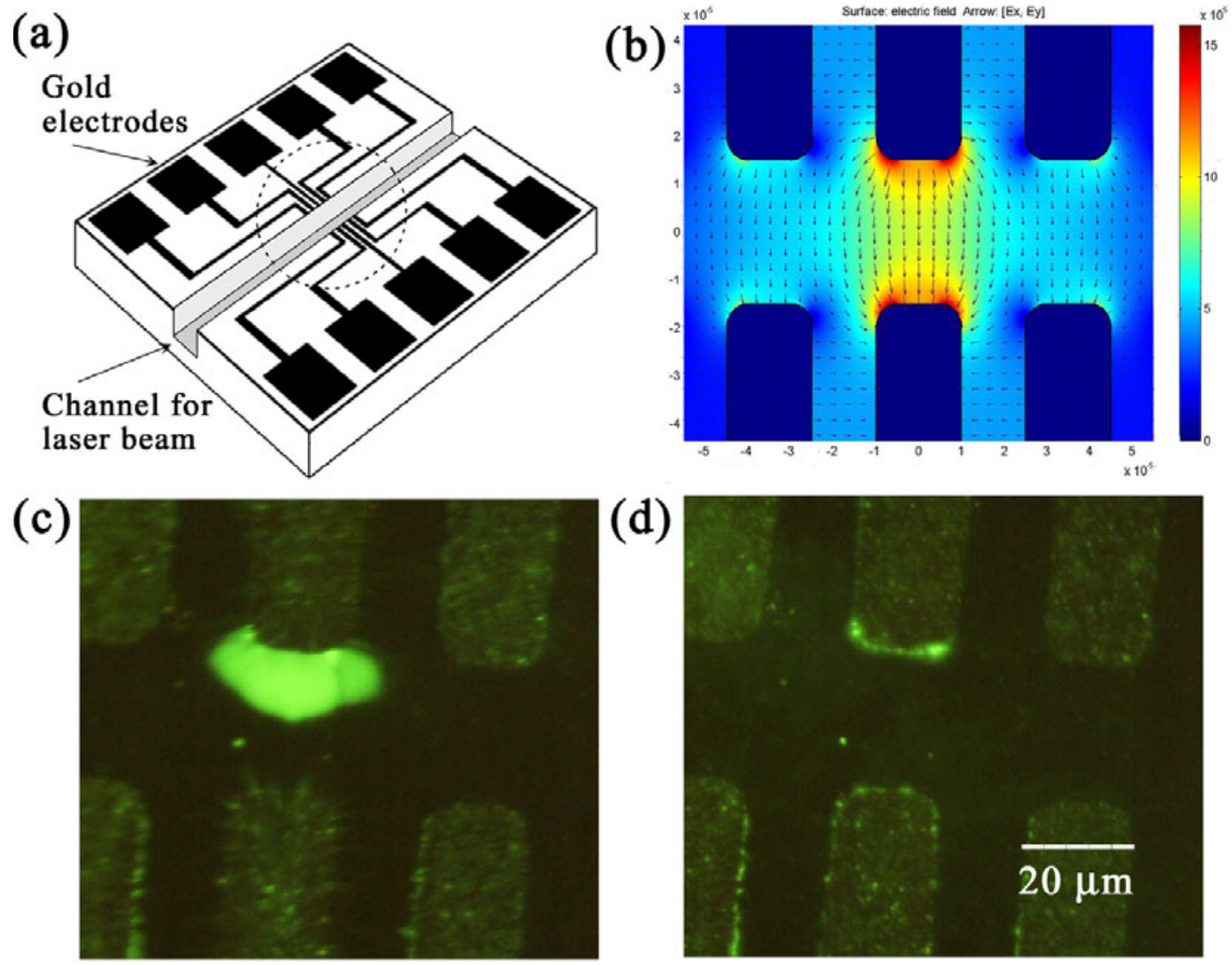

Figure 1. (a) Schematic view of the microelectrode set-up used. The laser beam is focused and directed into the channel for the laser-beam, which is indicated in the drawing. Figures $1(\mathrm{~b})-(\mathrm{d})$ are enlargements of the centre area of the electrode array indicated by the dotted curve. (b) The electric field was calculated using Femlab ${ }^{\circledR}$ software. The centre top electrode was set to a potential of $30 \mathrm{~V}$, the centre bottom electrode to $0 \mathrm{~V}$ and all other electrodes were left floating. The arrows indicate the direction of the electric field lines. (c) Fluorescence images of $\lambda$-DNA immobilized onto a $20 \mu \mathrm{m}$ wide electrode in the stretched and (d) in the relaxed state. The DNA was labelled using YOYO-1 and stretched using a $0.5 \mathrm{MV} \mathrm{m}^{-1}, 300 \mathrm{kHz}$ ac electric field.

and a large number of applications have emerged, including size determination of DNA, site-specific immobilization of DNA [6, 9], molecular surgery [6, 10], DNA sequencing [1113] and dielectric characterization of DNA fragments [14]. The use of microelectrodes allows the generation of the strong electric fields necessary for dielectrophoresis (of the order of $1 \mathrm{MV} \mathrm{m}^{-1}$ ) using relatively small potentials. It also facilitates the precise orientation and positioning of DNA at high spatial resolution. If tethered via a thiol group onto an electrode, DNA will be orientated and elongated out from the electrode when the electric field is applied and will return to its random coil conformation when the field is switched off, making it useful as a method to orientate specific DNA reversibly.

In this paper, we report a new method for the specific and controlled immobilization of DNA molecules $16 \mu \mathrm{m}$ in length to gold electrodes. We investigate the influence of the electric field frequency and amplitude on the dielectrophoresis of surface-bound $\lambda$-DNA molecules and show that fluorescentlylabelled $\lambda$-DNA molecules, tethered onto an array of electrodes using a multistep surface immobilization procedure, can be selectively addressed with an ac electric field. Furthermore, we demonstrate the use of dielectrophoresis to address surfacebound, fluorescently labelled DNA selectively in a basic datastorage device.

\section{Experimental details}

\subsection{Microelectrode device fabrication}

A series of electrodes was fabricated on an $\mathrm{Si} / \mathrm{SiO}_{2}$ wafer using standard UV photolithography, metal evaporation and lift-off. The electrodes comprised a $35 \mathrm{~nm}$ thick gold layer on a $10 \mathrm{~nm}$ thick $\mathrm{Ni} / \mathrm{Cr}$ adhesion layer and formed an opposing finger array pattern (figure 1). The electrodes were $10 \mu \mathrm{m}$ wide and $15 \mu \mathrm{m}$ apart with a 20 or $30 \mu \mathrm{m}$ gap between the tips of opposing electrodes. Following a second UV photolithography step, the $500 \mathrm{~nm}$ thick $\mathrm{SiO}_{2}$ layer between the opposing electrodes was removed using buffered HF for $8 \mathrm{~min}$. The remaining $\mathrm{SiO}_{2}$ layer was subsequently used as an etch mask to produce a $20 \mu \mathrm{m}$ deep groove in the underlying silicon layer by immersing the wafer in a $\mathrm{KOH} /$ propanol/ $/ \mathrm{H}_{2} \mathrm{O}$ solution for $25 \mathrm{~min}$ at $78^{\circ} \mathrm{C}$ and rinsing with deionized water. Prior to use, the wafer was cleaned by soaking in 'piranha' solution $\left(30 \% \mathrm{H}_{2} \mathrm{O}_{2}, 70 \% \mathrm{H}_{2} \mathrm{SO}_{4}\right)$ for $1 \mathrm{~h}$, rinsed in deionized water, ethanol, in deionized water again, and finally air dried. It has been reported that DNA can bind onto glass surfaces $[6,15]$, and so to prevent this, especially after the DNA is stretched, the exposed $\mathrm{SiO}_{2}$ surface was blocked by immersion in a $1: 5 \mathrm{v} / \mathrm{v}$ solution of trimethyl chlorosilane in tetrachloroethane for $1 \mathrm{~h} \mathrm{[16]} \mathrm{after} \mathrm{cleaning.} \mathrm{The} \mathrm{sample} \mathrm{was} \mathrm{then} \mathrm{rinsed} \mathrm{three}$ 
times with tetrachloroethane, followed by isopropanol, and thoroughly air dried. All chemical reagents were purchased from Sigma, unless otherwise noted.

\subsection{DNA immobilization}

$\lambda$-DNA is a long (48.5 kilobase pairs), double-stranded, circular DNA molecule, which has two single-strand nicks in the backbone, 12 bases apart. The circular conformation is maintained through these 12 Watson-Crick base pairs, which dissociate upon heating to produce a linearized DNA molecule with two single-stranded complementary ends, each 12 bases long. Thiolated $\lambda$-DNA did not adsorb directly from solution, even after $48 \mathrm{~h}$ incubation. Linearized $\lambda$-DNA adopts a random-coil conformation in solution [2], possibly reducing the probability of thiol-gold contact. To overcome this problem we developed the multi-step immobilization procedure outlined in figure 2 . The thiolated oligonucleotide, $\mathrm{X}$, of sequence $5^{\prime}$-AGGTCGCCGCCCTTTT-thiol-3' is, apart from the four $\mathrm{T}$ spacers, complementary to one of the singlestranded ends of the $\lambda$-DNA. The biotinylated oligonucleotide, Y, of sequence $5^{\prime}$-GGGCGGCGACCTTTTT-biotin-3' is, again apart from the four $\mathrm{T}$ spacers, complementary to $\mathrm{X}$ and therefore also complementary to the second $\lambda$-DNA end. Both oligonucleotides were $5^{\prime}$-phosphorylated and were purchased from MWG, Munich.

The oligonucleotides were hybridized to form a doublestranded molecule, $\mathrm{XY}$, in a $40 \mu \mathrm{l}$ solution by adding $2 \mu \mathrm{l} \mathrm{X}$ $(0.5 \mu \mathrm{M})$ and $2 \mu \mathrm{l} \mathrm{Y}(0.5 \mu \mathrm{M})$ to $36 \mu \mathrm{l}$ of TE $(10 \mathrm{mM}$ Tris- $\mathrm{HCl}$, $1 \mathrm{mMEDTA}, \mathrm{pH} 8.0), 1 \mathrm{M} \mathrm{NaCl}$ and incubating at $25^{\circ} \mathrm{C}$ for $1 \mathrm{~h}$. A $2 \mu \mathrm{l}$ droplet of this solution was placed on the electrode array, and incubated in a humid compartment at room temperature for $2 \mathrm{~h}$ during which the thiolated DNA adsorbed to the gold and became bound through the strong thiol-gold covalent bond (figure 2(a)) [2, 17]. The wafer was rinsed in deionized water for $5 \mathrm{~min}$ to remove unbound oligonucleotides and was then immersed in a $1 \mathrm{mM}$ 6-mercapto-1-hexanol $(\mathrm{MCH})$ solution for $1 \mathrm{~h}$ to block any regions of the gold electrodes not coated with XY (figure 2(b)) [3]. The oligonucleotides Y were then dissociated from the surface-bound oligonucleotides $\mathrm{X}$ by placing the wafer in a $70^{\circ} \mathrm{C}$ water bath for $10 \mathrm{~min}$, and were then rinsed in deionized water (figure 2(c)).

Each step in this immobilization procedure was separately validated on gold-coated microscope slides, using a biotin antibody immunodetection method that identifies the presence of the biotin on oligonucleotide Y [18]. The biotin is detected by binding to a biotin antibody, conjugated with alkaline phosphatase, which results in a colour change to dark blue when the antibody is stained using a solution of a Sigma Fast ${ }^{\mathrm{TM}}$ BCIP/NBT in deionized water. The colour change is quantified by measuring the reflectivity of the gold surface with a Perkin Elmer LS-50 luminescence spectrometer at $435 \mathrm{~nm}$ - higher surface coverage of biotinylated DNA leads to lower reflectivity of the gold surface after the immunodetection procedure [18]. The extent of XY adsorption was measured for different incubation times by this technique to establish that maximum surface coverage was achieved after $2 \mathrm{~h}$. Adsorption of XY was significantly reduced when the gold surface was previously covered by a $\mathrm{MCH}$ monolayer, showing that the $\mathrm{MCH}$ blocks DNA binding to gold. After the gold surface
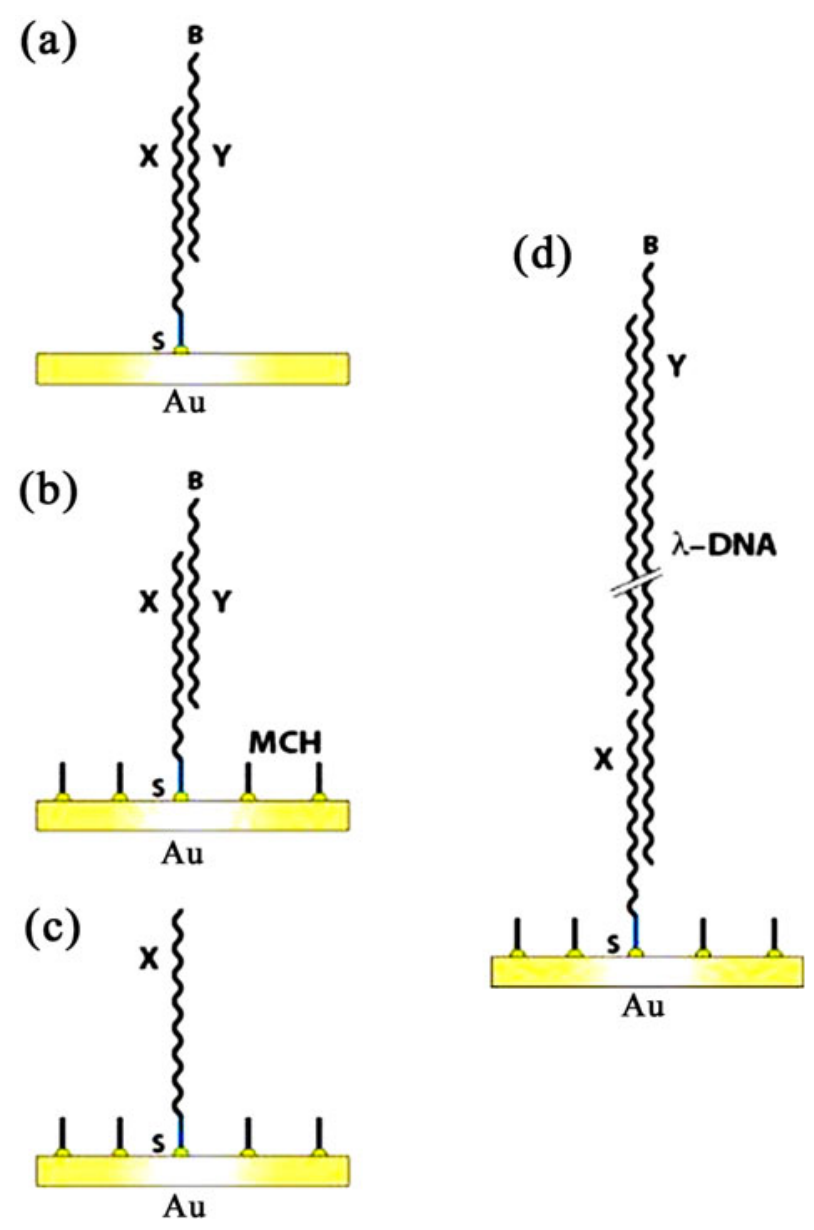

$\mathrm{Au}$

Figure 2. Schematic diagram showing the multistep procedure used to immobilize $\lambda$-DNA on the gold electrodes. Biotin is indicated by B and thiol by S. (a) Gold electrodes were coated with a monolayer of hybridized double-stranded oligonucleotides XY, followed by (b) immersing in 6-mercapto-1-hexanol (MCH) to block the surfaces not covered by $\mathrm{XY}$ and to orientate the oligonucleotides upwards. (c) Oligonucleotide $Y$ was dissociated by heating to $70^{\circ} \mathrm{C}$. (d) $\lambda$-DNA was then hybridized and subsequently ligated to the surface-bound oligonucleotide $\mathrm{X}$.

carrying the immobilized $\mathrm{XY}$ was incubated at $70^{\circ} \mathrm{C}$ for $10 \mathrm{~min}$, a subsequent immunodetection procedure revealed no biotin remaining on the surface, demonstrating that the biotinylated oligonucleotide $\mathrm{Y}$ had been removed.

One microlitre of a $1 \mu \mathrm{g} \mu \mathrm{l}^{-1}$ solution of $\lambda$-DNA was added to $9 \mu \mathrm{l} 1 \times \mathrm{TE}, 1 \mathrm{M} \mathrm{NaCl}$ and heated for $10 \mathrm{~min}$ to $65^{\circ} \mathrm{C}$ to linearize the molecules. To prevent the $\lambda$-DNA returning to its circular conformation, $2 \mu 1$ of $\boldsymbol{Y}$ was added to hybridize to the $\lambda$-DNA upon cooling. The linearized $\lambda$-DNA was labelled with a fluorescent intercalator, YOYO-1 (Molecular Probes), by adding $2 \mu 1$ of a solution of $2 \mu 1$ YOYO1 in $18 \mu \mathrm{l}$ phosphate buffer $\left(1.5 \mathrm{M} \mathrm{KH}_{2} \mathrm{PO}_{4}, \mathrm{pH}\right.$ 6.8) and incubating at $25^{\circ} \mathrm{C}$ for $10 \mathrm{~min}$. Unincorporated dye molecules and oligonucleotides were removed using a Microspin-400 column (Amersham Pharmacia Biotech). This corresponds to a molar ratio of intercalator to DNA base pairs of 1:8.

Two microlitres of the fluorescently labelled $\lambda$-DNA solution were applied to the wafer and incubated in a humid compartment at room temperature for $12 \mathrm{~h}$ to allow the remaining single-stranded end of the $\lambda$-DNA to hybridize to 


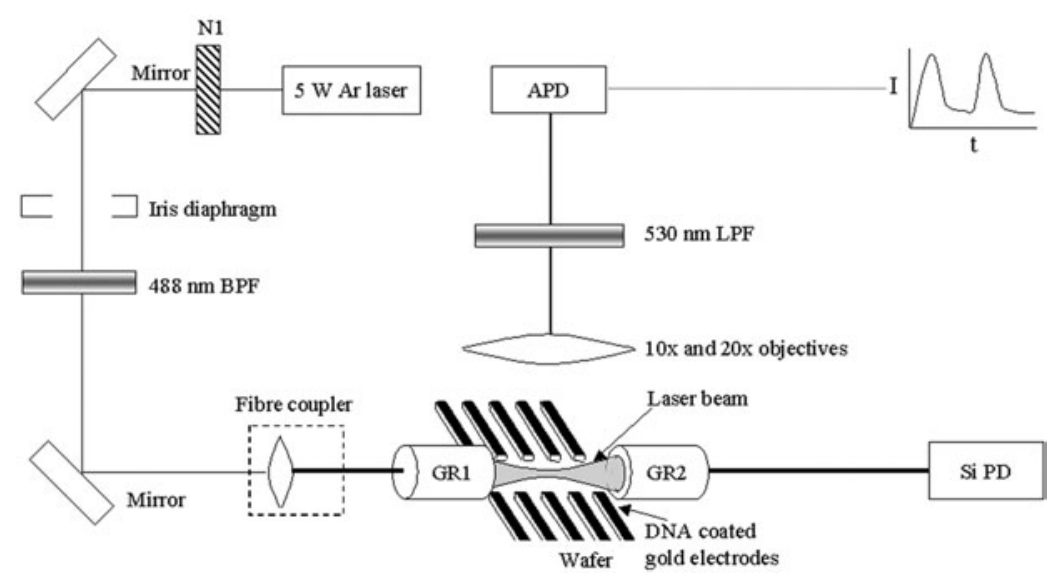

Figure 3. Schematic diagram of the optical set-up. The beam of a $5 \mathrm{~W}$ argon ion laser is attenuated with a neutral density filter (N1) and the $488 \mathrm{~nm}$ line was selected with a bandpass filter (BPF). The light is coupled into an optic fibre and focused into the groove on the wafer between the electrodes with a gradient-index micro lens (GR). At the other end of the groove, the light is collected with a second GR, coupled into a second fibre and the intensity measured with a silicon photodiode (Si PD). The fluorescence emission perpendicular to the surface was collected with a microscope-like combination of a $10 \times$ eyepiece and $20 \times$ objective, filtered, and its intensity measured with a silicon avalanche photodiode (APD).

the surface-bound oligonucleotide $\boldsymbol{X}$ (figure 2(d)). The wafer was then rinsed in deionized water for $5 \mathrm{~min}$. The surfacebound oligonucleotide $\boldsymbol{X}$ and the $\lambda$-DNA were ligated to form a single molecule by applying $2 \mu \mathrm{l}$ of a solution containing $1 \mu 110 \times$ ligation buffer and $1 \mu 1$ ligase (New England Biolabs) in $8 \mu \mathrm{l}$ deionized water, and incubating the wafer in a humid chamber for another $2 \mathrm{~h}$ at room temperature, followed by a final rinsing step with deionized water. Thereafter the wafer was kept submerged in deionized water. This hybridization and ligation process was verified by placing the wafer in a $70^{\circ} \mathrm{C}$ water bath for $10 \mathrm{~min}$ after which only ligated $\lambda$-DNA was expected to remain on the surface. The immunodetection procedure revealed the presence of $\mathrm{Y}$ when ligase was added, but not when the ligase was omitted.

\subsection{Optics set-up}

The optical arrangement is shown schematically in figure 3 . The beam of a $5 \mathrm{~W}$ argon ion laser was attenuated with a $1 \%$ transmission neutral density filter (N1), and scattered light excluded with an iris diaphragm. The $488 \mathrm{~nm}$ line (selected to correspond to the absorption maximum of the fluorophores at $491 \mathrm{~nm}$ ) was isolated with a bandpass filter (BPF) and coupled into a multimode fibre (0.48 numerical aperture). At the far end of the fibre, a 0.29 pitch gradient-index microlens (GR1) focused the light into the groove between the electrodes on the wafer, which was mounted on a high-precision $X Y Z$ and rotation stage to aid alignment. A second 0.29 pitch gradient-index microlens (GR2) coupled the light emerging from the groove into a second optic fibre for detection by a PIN photodiode for alignment purposes. The gradient index lenses and the wafer were submerged in deionized water. Fluorescence from the YOYO-1 was collected by a microscope-type combination of a $20 \times$ objective and a $10 \times$ eyepiece mounted above the wafer, and the intensity quantified with a silicon avalanche photodiode (APD) operated at room temperature at a reverse bias of $230 \mathrm{~V}$. A $530 \mathrm{~nm}$ long-pass filter (LPF) eliminated any scatter of the incident beam.
Dielectrophoretic stretching of the $\lambda$-DNA was achieved by applying an oscillating bias across two opposite electrodes (one grounded) using a $20 \mathrm{kHz}-1.1 \mathrm{MHz}$ signal generator. All other electrodes were left at a floating potential. The electric field referred to in this paper is the applied peak potential difference divided by the width of the gap between opposing electrodes. For all dielectrophoresis experiments, the DNA was suspended in deionized water (conductivity $5 \mu \mathrm{S} \mathrm{m}^{-1}$ ).

\section{Results and discussion}

\subsection{Dielectrophoretic stretching of surface-bound DNA}

Upon applying an ac electric field across two opposite electrodes, the DNA immobilized onto the electrodes stretched out to align parallel to the electric field lines. This alignment is due to the torque acting on the dominant induced dipole moment, which occurs axially along the DNA [10-12], and forms a band-like structure around the electrodes (figure 1). In terms of the dipole moment, $\boldsymbol{p}$, and the electric field, $\boldsymbol{E}$, the torque experienced by the DNA is given by $\boldsymbol{T}=\boldsymbol{p} \times \boldsymbol{E}$. The dipole moment depends on the permittivity of the liquid and the DNA, respectively, as well as on the electric field, $\boldsymbol{E}$, and the length of the DNA [19]. We note that the torque depends on the electric field vector, not its gradient, and therefore a torque is exerted on a dipole even in a uniform field. The torque results in an orientation of the dipole relative to the electric field lines until $\boldsymbol{p}$ and $\boldsymbol{E}$ are parallel [8].

In contrast to many dielectrophoresis experiments in which the DNA is free in solution [4-6, 9-13], here the DNA is tethered to a surface by strong thiol-gold bonds. The average maximum length of the elongated DNA depends on the frequency and the magnitude of the ac field [4]. In most applications involving dielectrophoretic manipulation of molecules, the dielectrophoresis needs to be optimized with respect to amplitude and frequency of the applied electric field. Therefore, we have investigated the length of the elongated DNA, which was determined by measuring the width of the fluorescent band around the electrodes (see figure 1(c)), 


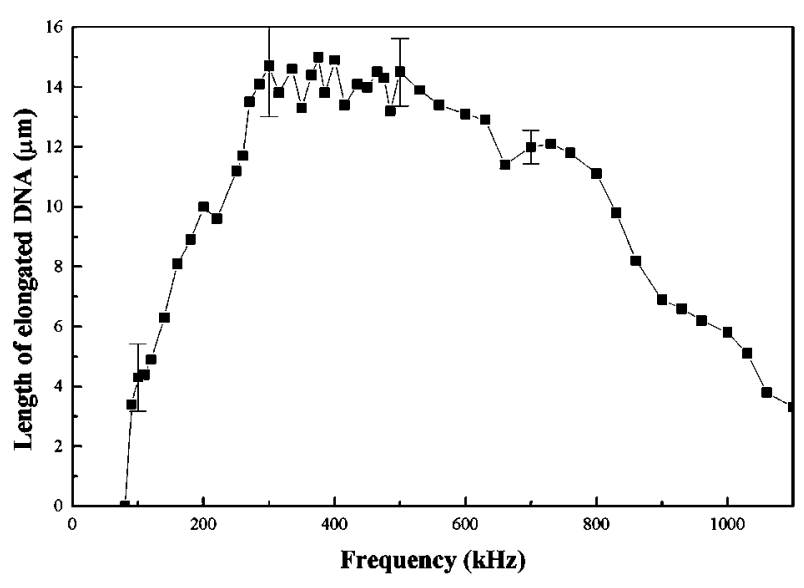

Figure 4. Length of the elongated $\lambda$-DNA upon dielectrophoretic stretching as a function of frequency under a $15 \mathrm{~V}$ potential across a $30 \mu \mathrm{m}$ gap. The $\lambda$-DNA was immobilized onto the gold electrodes using the multistep procedure and the length of the DNA was determined by measuring the distance between the electrode edge and the end of the fluorescence band (see figure 1(c)). The data represent an average of three measurements and the error bars indicate the standard deviation.

as a result of dielectrophoretic orientation and elongation as a function of frequency, and the results are shown in figure 4. The length of the elongated DNA decreases with increasing frequency above $100 \mathrm{kHz}$ owing to the decreasing dielectrophoretic force and torque, which is due to the decrease of the induced dipole. The reorientation of the dipole on the DNA starts lagging behind the switch in the polarity of the electrodes in the ac field, therefore reducing its size in the frequency range from $100 \mathrm{kHz}$ to $1 \mathrm{MHz}$. The magnitude of the dielectrophoretic force is therefore expected to decrease over this region [14]. Below about $100 \mathrm{kHz}$ there is a change in the DNA polarization and the band collapses onto the electrode, as observed in [6]. The natural contour length of $\lambda$-DNA is $L_{0}=16.5 \mu \mathrm{m}$ [20], but the high concentration of the intercalated fluorophores causes an expansion of the DNA molecule to length $L=L_{0}(1+2 r)$, where $r$ is the molar ratio of intercalator to base pairs ( $r=1 / 8$ in this case) [21]. Thus, the length of fully stretched YOYO-1-labelled $\lambda$-DNA is about $21 \mu \mathrm{m}$. However, we observed a maximum length of only about $15 \mu \mathrm{m}$, which suggests that although a maximal stretching was reached at frequencies of around $300-500 \mathrm{kHz}$ (figure 4), the DNA was not fully elongated. In this range the force on the DNA reaches a maximum as the dipole moment on the DNA decreases with an increase in frequency, but is still not strong enough to fully extent the DNA.

Dielectrophoretic forces exist in nonuniform ac and dc electric fields. We employed ac electric fields to generate the dielectrophoretic forces on the DNA because dc fields lead to electrolytic dissociation of the aqueous medium, even at low potentials. The electric field range, over which the DNA is stretched and orientated, is limited to about 0.2 to $0.8 \mathrm{MV} \mathrm{m}^{-1}$. At lower electric fields the dielectrophoretic force is dominated by thermal randomization of the orientation of the DNA, and no alignment of the DNA can be observed. Higher electric fields result in stronger forces, but at low frequencies (below about $100 \mathrm{kHz}$ ) electrothermal reactions start taking place. Joule heating results in local density, viscosity, permittivity

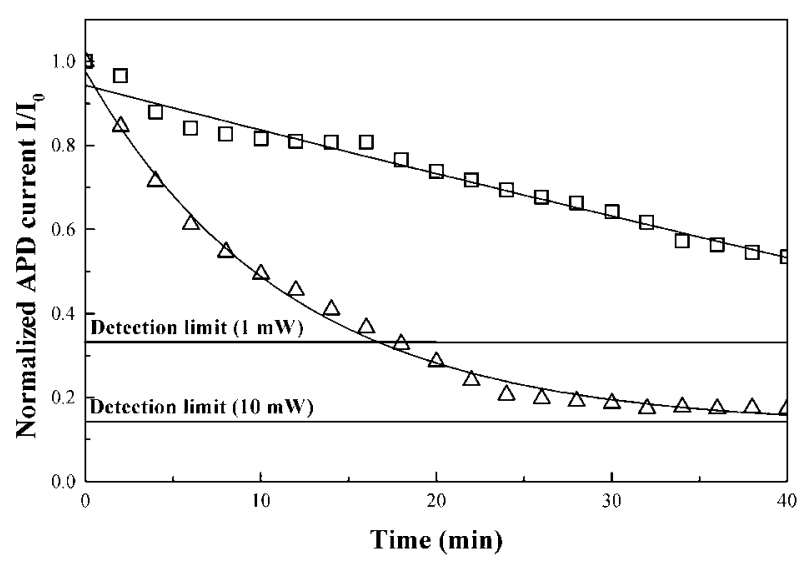

Figure 5. Normalized fluorescence intensity of the

YOYO-1-labelled DNA as a function of illumination time under $1 \mathrm{~mW}$ (squares) and $10 \mathrm{~mW}$ (triangles) laser power. The background signal has been subtracted. The lines are first-order exponential decay fits. Only one electrode was addressed in each experiment.

and conductivity changes. A dielectric force exists across a permittivity gradient and a Coulomb force across a conductivity gradient, and these frequency-dependent electrothermal forces result in fluid flow [22], which can disrupt the stable orientation of the DNA. At high frequencies a higher voltage can be applied before electrothermal forces are observed.

As a control, only one electrode of the array was coated with $\lambda$-DNA. Applying an electric field between neighbouring uncoated electrodes produced no observable effect on the $\lambda$-DNA on a neighbouring electrode and no accumulation of DNA on the clean, biased electrodes was observed. This indicates that the DNA was firmly attached on the coated electrode and that the multistep immobilization procedure leads to a stable medium.

\subsection{A possible application: basic data-storage device}

Increased demands for data-storage capacity are focusing research attention on the improvement of existing datastorage media and the development of alternative data-storage systems [23]. DNA is increasingly being investigated as a possible storage medium, due to its natural ability to encode biological data and its unique physico-chemical self-assembly capability. At present the use of sequence information for data storage is limited by the difficulty in reading and writing data using slow PCR and sequencing techniques [24]. To overcome this rate limitation, the unique physical properties of DNA are increasingly being exploited. DNA has, for example, been used as a scaffold for the assembly of fluorescent resonance energy transfer donors and acceptors [25]. The fluorescence is switched from an 'on' to an 'off' state by adding quencher groups, and reading of the data points is based on laser excitation. This type of approach has the advantage of being volumetric, in that multiple donor-acceptor pairs can be arrayed along the length of a single DNA chain. Here, we investigate an alternative approach for using DNA in data storage devices. Our concept is to reversibly orientate and elongate fluorescently labelled DNA into a read-write laser beam using dielectrophoretic manipulation. The data are encoded by selective photobleaching of the fluorophores. 
Selective dielectrophoretic manipulation of surface-immobilized DNA molecules

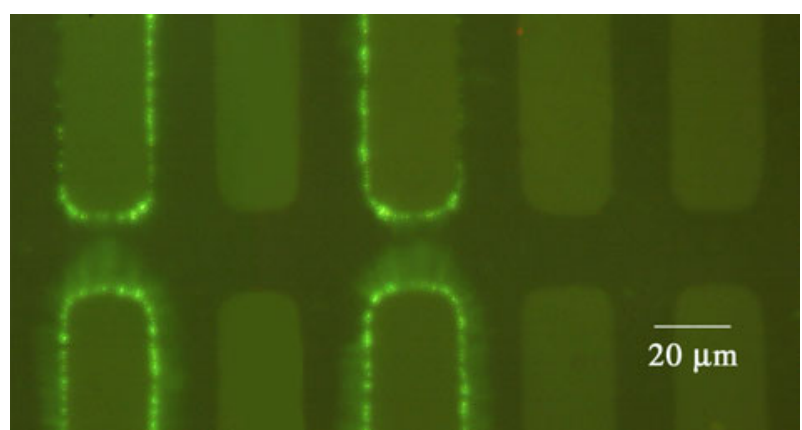

Figure 6. Fluorescence image of an array of five gold electrodes at the beginning of the writing step. The $\lambda$-DNA on the first and third electrode is selectively stretched into the $10 \mathrm{~mW}$ laser beam by a $0.5 \mathrm{MV} \mathrm{m}^{-1}, 400 \mathrm{kHz}$ ac electric field to be photobleached. The photobleaching was complete after 30 min thus leading to a stored binary pattern 10100 . The image was acquired with a cooled CCD camera with a $525 \mathrm{~nm}$ bandpass filter.

In order to use this system as a basic data-storage device, an electrode array was coated with fluorescently labelled $\lambda$-DNA using a multistep immobilization procedure (see Materials and Methods). Information was stored by photobleaching the fluorescent DNA attached to specific selected electrodes, following dielectrophoretic elongation of the selected DNA 'bit'. Only the fluorophores intercalated into the DNA molecules reaching the laser beam were photobleached and subsequently detected in the reading step. An electric field of $0.5 \mathrm{MV} \mathrm{m}^{-1}$ at $400 \mathrm{kHz}$ was applied to ensure that the DNA orientated into the groove was maximally elongated (see figure 4).

Figure 5 graphs the intensity of the emitted light from the fluorophores excited by a $10 \mathrm{~mW}$ laser beam as a function of exposure time. The laser power was measured at the point before the light was coupled into the fibre (see figure 3). The emitted intensity decreased with time due to ongoing photobleaching, and reached the detection limit of the photodiode after about $30 \mathrm{~min}$. This extended 'write' time suggests good stability of the encoded information during subsequent reading at lower laser intensity. Increasing the laser power would decrease the photobleaching time, but can also result in DNA photocleavage [26, 27]. For the molar ratio of intercalator to base pairs used in this study (1:8), the YOYO-1 fluorophores are bis-intercalated into the base pairs of the DNA [26], and are photolysed by $\cdot \mathrm{OH}$ radicals when illuminated with intense light causing singlestranded breakage of the DNA. The fluorophores do not cleave the double-stranded DNA, unless two single-stranded breaks occur less than 15 base pairs apart [26]. Cleavage of the $\lambda$-DNA causes free DNA fragments in solution, which, in this data-storage application, will lead to an increase of the background signal with time. This may lead to false signals if a photobleached electrode was addressed and fluorescent DNA fragments in solution were attracted towards it. However, we experienced no significant photocleavage even when using a $10 \mathrm{~mW}$ beam. Figure 6 shows an array of five electrodes in which the fluorescently labelled $\lambda$-DNA on the first and third electrodes have been simultaneously orientated and elongated in preparation to being photobleached for $30 \mathrm{~min}$ in the $10 \mathrm{~mW}$ laser beam. This will write a binary 10100, where

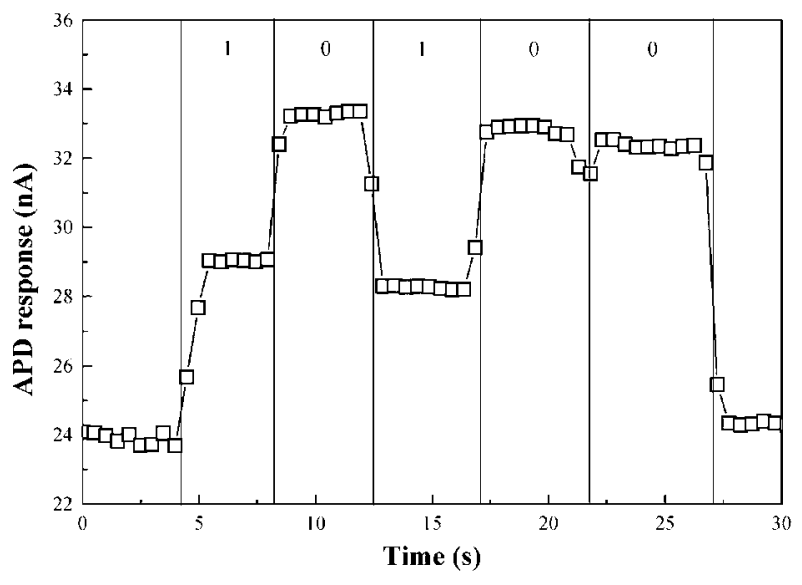

Figure 7. The response of the APD as a function of time while addressing the electrodes of the array sequentially for $5 \mathrm{~s}$ each. Low APD currents correspond to low fluorescence intensities and therefore to binary 1's, whereas high currents correspond to high fluorescence intensities and to binary 0's. A laser power of $1 \mathrm{~mW}$ was used in the reading step.

a photobleached electrode corresponds to a binary ' 1 ', and a fluorescent electrode corresponds to a binary ' 0 '.

The reading procedure consists of briefly elongating the $\lambda$-DNA on each electrode sequentially into the laser beam and measuring the fluorescence intensity. A reduced laser power of $1 \mathrm{~mW}$ is used which, as can be seen from figure 5, produces a fluorescence intensity of well above the detection limit. The photobleaching time at this low power is substantially longer than 30 min (figure 5) indicating that the reading procedure does not significantly bleach fluorescent electrodes. Figure 7 shows the response of the APD as a function of time in which the electrodes were addressed sequentially for about $5 \mathrm{~s}$ each, which reveals the binary sequence 10100 that was written originally. The fluorescence intensity of both the photobleached and intact fluorescent $\lambda$-DNA remained stable over a period of at least 2 days, and reversible elongation also remained possible over this period.

The basic data-storage application discussed above is only a proof-of-concept study and it is possible to envisage the use of the dielectrophoretic addressing mechanism in conjunction with AFM to manipulate short DNA strands immobilized on a surface. It has been shown that long DNA molecules can be orientated with dielectrophoresis using an AFM tip [28]. A possible route to increase the recording performance may be the use of photoactivatable green fluorescent protein (GFP) that can be optically switched with $413 \mathrm{~nm}$ light to fluorescence 100 times more intense when excited by $488 \mathrm{~nm}$ light [29]. In this set-up the DNA is immobilized on a metal surface and surrounded by quencher groups to prevent photoactivation. The selected DNA is pulled out of this quenching environment with high spatial resolution into the $413 \mathrm{~nm}$ irradiation where it is rapidly switched, leading to a pattern of high and low fluorescence.

\section{Conclusions}

We have demonstrated a multistep surface immobilization procedure, which allows the specific and controlled immobilization of long DNA molecules onto gold electrodes. The gold electrodes are functionalized with short 
single-stranded DNA oligonucleotides bound to the surface via a thiol group. We showed that long DNA molecules with a single-stranded overhang complementary to the surface-bound oligonucleotide are hybridized and ligated onto the surface with an efficiency substantially greater than that achieved by direct immobilization procedures.

$\lambda$-DNA immobilized on gold electrodes has been aligned and elongated by an ac electric field applied across two opposing electrodes. We have determined the length of the elongated DNA and found that a maximum length was achieved at about 300-500 kHz. This maximum length of approximately $15 \mu \mathrm{m}$ suggests that the DNA was not fully extended when compared with the theoretical length of the YOYO-intercalated $\lambda$-DNA $(21 \mu \mathrm{m})$.

Finally, we have discussed a possible application capitalizing on the results of this work, namely a basic data-storage device using fluorescently labelled $\lambda$-DNA, immobilized onto an array of gold electrodes. The DNA molecules on each electrode were elongated selectively into a focused laser beam using the dielectrophoretic addressing mechanism. Data were written into the medium by selectively elongating DNA into a $10 \mathrm{~mW}$ laser beam for $30 \mathrm{~min}$, leading to photobleaching of the intercalated fluorophores. Subsequent reading of the stored data uses the same addressing mechanism but is performed at lower laser intensity. We showed that this dielectrophoretic addressing mechanism, together with photobleaching of the YOYO-1 fluorophores, is a reliable method of writing and reading data onto the DNA.

\section{Acknowledgments}

We thank $\mathrm{S} S$ Dhillon and $\mathrm{R}$ Bates for their help with the chip processing. This research was funded in part by the EPSRC. AGD, WAG and CW acknowledge the Royal Society, the Cambridge Commonwealth Trust, and the Schweizerische Nationalfonds zur Förderung der Wissenschaftlichen Forschung, respectively. RW thanks the Gottlieb Daimler and Karl Benz-Stiftung and the George and Julian Schiff Foundation.

\section{References}

[1] Chen Y and Pépin A 2001 Electrophoresis 22187

[2] Steel A B, Levicky R L, Herne T M and Tarlov M J 2000 Biophys. J. 79975
[3] Levicky R L, Herne T M, Tarlov M J and Satija S K 1998 J. Am. Chem. Soc. 1209787

[4] Asbury C L and Van den Engh G 1998 Biophys. J. 741024

[5] Suzuki S, Yamanashi T, Tazawa S, Kurosawa O and Washizu M 1998 IEEE Trans. Ind. Appl. 3475

[6] Washizu M, Kurosawa O, Arai I, Suzuki S and Shimamoto N 1995 IEEE Trans. Ind. Appl. 31447

[7] Pohl H A 1978 Dielectrophoresis (Cambridge: Cambridge University Press)

[8] Gimsa J 2001 Bioelectrochemistry 5423

[9] Washizu M and Kurosawa O 1990 IEEE Trans. Ind. Appl. 26 1165

[10] Yamamoto T, Kurosawa O, Kabata H, Shimamoto N and Washizu M 2000 IEEE Trans. Ind. Appl. 361010

[11] Kabata H, Okada W and Washizu M 2000 Japan. J. Appl. Phys. I 397165

[12] Kawabata T and Washizu M 2001 IEEE Trans. Ind. Appl. 37 1625

[13] Oana H, Ueda M and Washizu M 1999 Biochem. Biophys. Res. Commun. 265140

[14] Bakewell D J, Morgan H and Milner J J 1999 Characterisation of the dielectrophoretic movement of DNA in micro-fabricated structures 10th Int. Conf. on Electrostatics (Cambridge) (Bristol: Institute of Physics Publishing)

[15] Allemand J F, Bensimon D, Jullien L, Bensimon A and Croquette V 1997 Biophys. J. 732064

[16] Braun E, Eichen Y, Sivan U and Ben-Yoseph G 1998 Nature 391775

[17] Esplandiú M J, Hagenström H and Kolb D M 2001 Langmuir 17828

[18] Wirtz R, Wälti C, Germishuizen W A, Pepper M, Middelberg A P J and Davies A G 2003 Nanotechnology 147

[19] Jones T B 1995 Electromechanics of Particles (Cambridge: Cambridge University Press)

[20] Maier B and Rädler O 2000 Macromolecules 337185

[21] Daune M 1999 Molecular Biophysics-Structures in Motion (Oxford: Oxford University Press)

[22] Ramos A, Morgan H, Green N G and Castellanos A 1998 J. Phys. D: Appl. Phys. 312338

[23] Hosaka S, Kikukawa A, Koyanagi H, Shintani T, Miyamoto M, Nakamura K and Etoh K 1997 Nanotechnology 8 A58

[24] Calude C S and Casti J L 1998 Nature 392549

[25] Heller M J and Tu E 1994 US Patent Specification 5787032

[26] Kanony C, Akerman B and Tuite E $2001 \mathrm{~J}$. Am. Chem. Soc. 1237985

[27] Akerman B and Tuite E 1996 Nucl. Acids Res. 241080

[28] Ueda M, Baba Y, Iwasaki H, Kurosawa O and Washizu M 1999 Japan. J. Appl. Phys. 386568

[29] Patterson G H and Lippincott-Schwartz J 2002 Science 297 1873 